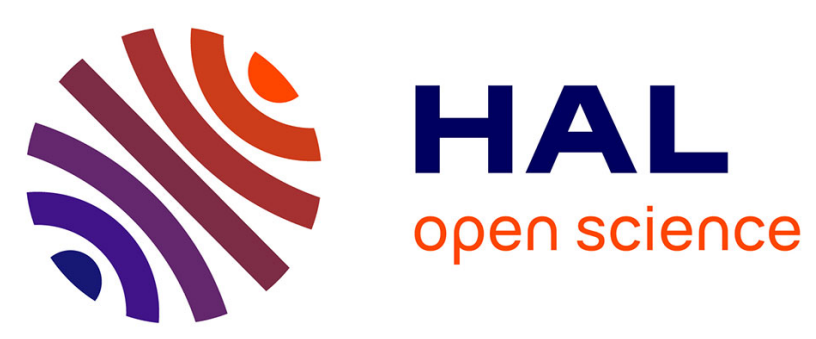

\title{
Advantages and limitations of virtual reality for balance assessment and rehabilitation
}

Marion Morel, Benoit Bideau, J. Lardy, Richard Kulpa

\section{To cite this version:}

Marion Morel, Benoit Bideau, J. Lardy, Richard Kulpa. Advantages and limitations of virtual reality for balance assessment and rehabilitation. Neurophysiologie Clinique $=$ Clinical Neurophysiology, 2015, 45 (4-5), pp.315-326. 10.1016/j.neucli.2015.09.007 . hal-01225506

\section{HAL Id: hal-01225506 \\ https://hal-univ-rennes1.archives-ouvertes.fr/hal-01225506}

Submitted on 13 Jan 2016

HAL is a multi-disciplinary open access archive for the deposit and dissemination of scientific research documents, whether they are published or not. The documents may come from teaching and research institutions in France or abroad, or from public or private research centers.
L'archive ouverte pluridisciplinaire HAL, est destinée au dépôt et à la diffusion de documents scientifiques de niveau recherche, publiés ou non, émanant des établissements d'enseignement et de recherche français ou étrangers, des laboratoires publics ou privés. 


\title{
Advantages and limitations of virtual reality for balance assessment and rehabilitation
}

\section{Avantages et limites de la réalité virtuelle pour l'évaluation et la rééducation de l'équilibre}

\author{
M. Morel ${ }^{1,2}$, B. Bideau ${ }^{1}$, J. Lardy ${ }^{1}$, R. Kulpa ${ }^{1}$ \\ ${ }^{1} \mathrm{M} 2 \mathrm{~S}$ laboratory - University of Rennes 2 \\ ENS Rennes - Campus de Ker Lann - Avenue Robert Schuman, 35170 Bruz \\ ${ }^{2}$ M2S laboratory - University of Rennes 2 \\ ENS Rennes - Campus de Ker Lann - Avenue Robert Schuman, 35170 Bruz
}

\section{Auteur référent}

Marion Morel

Tel : 0642344888

Email : marion.morel@ens-cachan.fr

\section{Summary}

Virtual Reality (VR) is now commonly used in many domains because of its ability to propose a standardized, reproducible and controllable environment. In balance assessment, it can be used to control the stimuli presented to the patients and thus accurately evaluate their evolution or compare them to different populations in standardized situations. In balance rehabilitation, VR allows the creation of new generation of tools and at the same time the way to assess the efficiency of each parameter of these tools to optimize them. Moreover, with the development of low-cost devices, this rehabilitation can be continued at home, easing the access to these tools, in addition to their ludic and thus motivating properties. Nevertheless, and even more with low-cost systems, VR has limits that can alter the results of the studies that use it: the latency of the system (the delay cumulated on each step of the process from data acquisition on the patients to multimodal outputs) and the perception of distances that is underestimated in VR. After having described why VR is an essential tool for balance assessment and rehabilitation and illustrated this statement with a case study, this review links the previous works on that domain with the technological limits of VR.

\section{Keywords}

Virtual reality, balance, assessment, rehabilitation

\section{Résumé}

La Réalité Virtuelle (RV) est utilisée dans de nombreux domaines puisqu'elle permet d'avoir un environnement standardisé, reproductible et contrôlable. Pour l'évaluation de l'équilibre, elle permet de contrôler les stimuli visuels proposés aux patients et donc d'évaluer précisément leur évolution ou de les comparer à d'autres populations dans un environnement standardisé. Pour la rééducation de l'équilibre, la RV permet la création d'une nouvelle génération d'outils et en même temps le moyen d'évaluer chacun des paramètres de ces outils pour les optimiser. De plus, avec le développement des dispositifs bas coût, la rééducation peut se poursuivre à domicile, rendant les outils plus accessibles, en plus de leur aspect ludique et donc motivant. Néanmoins, et particulièrement avec les systèmes bas coût, la RV possède des limites qui peuvent altérer les résultats des études qui l'utilisent: la latence du système (le délai cumulé par 
toutes les étapes du processus allant de l'acquisition des données des patients jusqu'au rendu multimodal) et la perception des distances qui est sous-estimée en RV. Après avoir décrit les raisons pour lesquelles la RV est un outil essentiel pour l'évaluation et le rééducation de l'équilibre et l'avoir illustré avec un cas d'étude, cette revue fait la correspondance entre les études de la littérature dans ce domaine et les limites technologiques de la RV.

\section{Mots-clés}

Réalité virtuelle, équilibre, évaluation, rééducation

\section{$1 \quad$ Introduction}

Virtual reality (VR) is now commonly used in many domains such as for the training of aircraft pilots, or of workers ensuring the maintenance of nuclear sites for instance, both for security and economic reasons. Similarly, in the medical field, VR has been used for the training of surgeons, especially for laparoscopic surgery, or for the treatment of phobias for example. The secure environment allows to control the stimuli presented to the patient so he can face his fear gradually. In addition to the secure aspect, VR provides a standardized and controlled environment that allows analyses and experiments that cannot be done in real situation. It is the reason why clinical assessment and rehabilitation has studied the potential advantages of incorporating VR technologies into patient training for many years.

Recently, video games industry have made strong advancement in the creation of low-cost VR systems such as Microsoft Kinect (Microsoft Corp., Redmond, Washington) or Nintendo Wii (Nintendo Co. Ltd., Kyoto, Japan). But the emergence of crowdfunded products, such as Oculus Rift, Razor Hydra or Perception Neuron, all financed by the Kickstarter company, is even more accelerating the use of VR for rehabilitation. Levac et al. have even proposed resources to support decision making about integration of Kinect into rehabilitation clinical practice [1].

Many companies besides offer training tools for the general public: the exergames. They allow the patient to continue their rehabilitation at home based on such low-cost devices. But even if VR seems to be considered as the promising tool for clinical assessment and rehabilitation because of its many advantages, it has a number of limitations that are sometimes omitted or understated. This is the reason why this paper is discussing about how VR can be really useful for balance rehabilitation and how it should be setup.

\section{$2 \quad$ Why Virtual Reality for balance assessment and rehabilitation?}

\section{$2.1 \quad$ Case study of pendulums for balance assessment}

Many protocols can be set up to evaluate the balance recovery of patients. As soon as you can disturb his/her balance for instance, you can analyze the way the patient recovers its equilibrium. Nevertheless, it is necessary to have a standardized and reproducible environment to compare the trials of a patient (evolution of his balance in function of time, training sessions, etc.) or between patients. In several domains, VR has exhibited better performance than video such as in laparoscopic surgery [2] or in physical activity [3]. So why VR can be so pertinent for balance assessment and rehabilitation?

Let consider the training of balance recovery of elderly people with the use of a system composed of three pendulums. The objective of such a system is to slowly throw balls toward the head of the patient forcing him to avoid collision and then to recover balance. The three balls ensure uncertainty from where the ball is coming to avoid anticipation. 
This system can only be usable if two main issues are solved. First, it must be adapted to the different morphologies of patients. The balls must indeed go toward the head whatever the size of the patient. Second, the speed of the balls must be controlled since it depends on the ability of the patient to quickly avoid the ball or not. It can be related to the age or the physical fitness of the patient for instance.

The first solution is to create a real structure in which the wires of the pendulum must be adaptable to control the trajectory of the balls (see figure 1a). It can be achieved by adjusting 1 ) the height of the spire on which the wires are tied to deal with the patient's morphology, 2) the starting position of the balls and the length of the wires to change the speed of the balls.

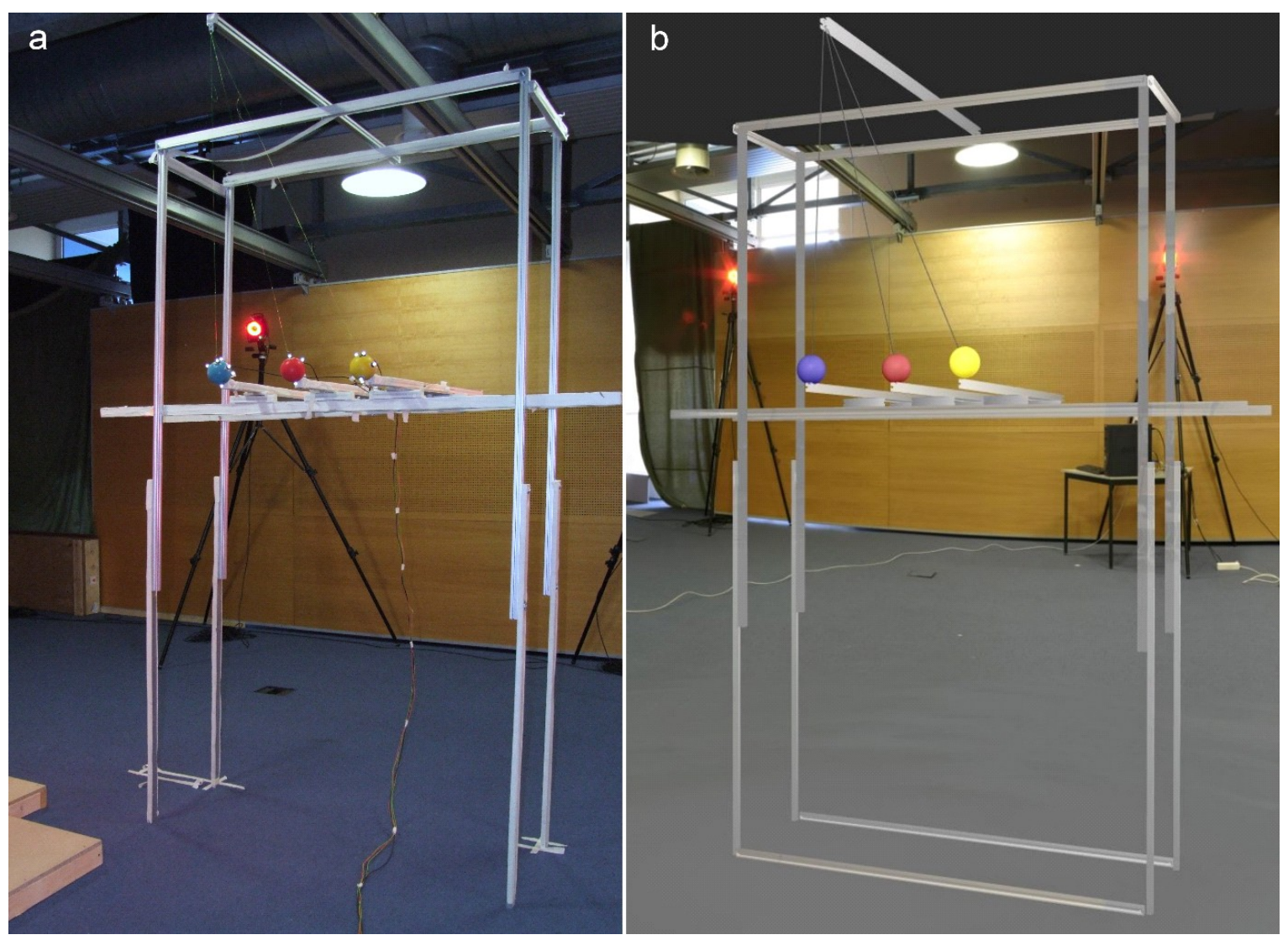

Figure 1: Case study setup. a) Real structure composed of three pendulums. It can be manually adjusted to the patient's height and to control the orientation and speed of the balls. b) Same structure in a virtual environment. It can be automatically adjusted to the patients and to simulation parameters.

Besides the cost of the development and conception of such a system, two main problems arise. The space needed to install this structure is often incompatible with doctor's office. But more problematic is the parameterization of the system. For instance, to modify the height of the structure, it is needed to unscrew, move and screw again the metallic bars. But far more complex is to determine the configuration of the structure since the height of the spire also depends on the length of the wires that depends on the desired balls' speed which also depends on the starting position of the balls. This kind of system is then not suitable for the training of several patients.

The second solution is to create a virtual representation of this structure and to use VR to control its simulation and immerse the patient in front of it (see figure $1 \mathrm{~b}$ ). Several steps are then required: 1 ) create the virtual environment, 2) create the kinematic simulation of the pendulums, 3) handle the inputs (head tracking and force plate) and output (visual feedback). In this virtual environment, a model of movement of the balls can be computed to propose an automatic adaptation of all the structure directly from some parameters such as the patient's height and the ball speed. 
With the emergence of new low-cost devices, this kind of system can be used in doctor's surgery, for example by combining a Microsoft Kinect to capture the motion of the patient, an Oculus Rift to immerse the subject into the virtual environment with a stereoscopic vision while the embedded head tracking in rotation allows a field of regard of 360 degrees. Finally, a Nintendo Wii Balance Board can be used to acquire the center of pressure displacement.

This case study shows how VR can provide a standardized and reproducible environment that allows the analysis and rehabilitation of balance. Nevertheless, the same way it reveals strong advantages, it has some limits that can alter the results obtained with such a system.

\subsection{Advantages and limits of VR}

Many studies are based on VR, it goes from experiment with projection of 3D objects on a monoscopic screen up to the immersion of the subject with multi-sensory feedbacks (visual, tactile, and auditory for example). Such a large disparity in studies is explained because there is not a single definition of VR. The most common definition is that VR is a scientific and technical domain that exploits computer sciences and behavioral interfaces to simulate in a virtual world the behavior of 3D entities that interact with each other in real time and with users immersed in a pseudo-natural manner through sensorimotor channels. A VR system is efficient when the user has the "feeling of being there" (in the virtual world) [4, 5]. This is the concept of presence [6].

As stated above, the first advantage of VR is the complete control of the stimuli provided to the subject. It is the main reason to use VR because it provides a standardized and reproducible environment [7]. The second advantage is the ability to have stereoscopic vision that gives the subject salient motion-in-depth information [8]. Moreover, perspective in a virtual environment can be adapted in real-time to correspond to the subject's viewpoint. In addition to these advantages, VR is often seen as a fun training tool increasing the motivation of patients to continue their rehabilitation.

Nevertheless, all these advantages are obtained thanks to software computations and hardware devices. All the steps from the capture of information (motion, center of pressure, etc.) to the multisensory feedback (at least visual one) takes time and the delay can be perceived by the immersed patient and can modify his reactions. This is called latency of the system. The second main limit of VR is the different perception of distances. The latter are indeed underestimated in VR compared to real situation. Depending on the type of study, it is thus necessary to take into account these limits that are often ignored or understated. They can indeed alter the actions of the patients who can have different amplitude of center of pressure or reaction times in VR compared to real situation for instance.

\subsection{Evaluation of the case study between real and virtual environments}

To evaluate the relevance of using VR for balance assessment or rehabilitation, we have made an experiment based on the case study described above with the three pendulums. The objective was to compare the reaction of the subject in real and in VR during balance recovery, as it has been done for example in sports where the reactions of a goalkeeper in front of a real kicker was compared to the same situation in VR in front of the virtual kicker [9]. Nevertheless, in such a study, only the kinematics of the subject is analyzed. When dealing with balance assessment and rehabilitation, studying the motion dynamics is essential.

The experiment proposed three different conditions. The first one is the reference condition since the subject is placed in front of the real structure and has to avoid the balls (see figure 1). The second condition evaluates the influence of having a head-mounted display (HMD) device only. The HMD can indeed influence the motion dynamics of the subject by its weight or by altering the way he moves, for instance due to the wire connecting the HMD to the computer. We thus used a special see-through HMD which can be used as glasses if no images are projected (Visette 45 SXGA, Cybermind, dedicated to virtual and augmented reality). Finally, the third condition is the immersion of the subject in the VR environment presented in figure 2 thanks to the HMD without the see-through option. 
In these three conditions, 18 healthy subjects have to simply avoid the coming balls that are thrown one at a time in a randomized order to avoid anticipation, real balls for the two first conditions and virtual balls for the third one. Each trial is repeated 10 times. Data collected were the center of pressure (CoP) displacement and the ground reaction forces (GRF).

As described in figure $2 \mathrm{a}$, the results first showed that the response time of the subject was significantly delayed in VR compared to the two real situations (with or without HMD). This delay can logically be observed in the first variation of CoP and the evolution of ground reaction forces. It is quite constant since its value is $270 \pm 5 \mathrm{~ms}$. It is obviously a consequence of the latency of the system but it can also be due to the perception of distance that let the subjects consider that they are more time to react than in real.
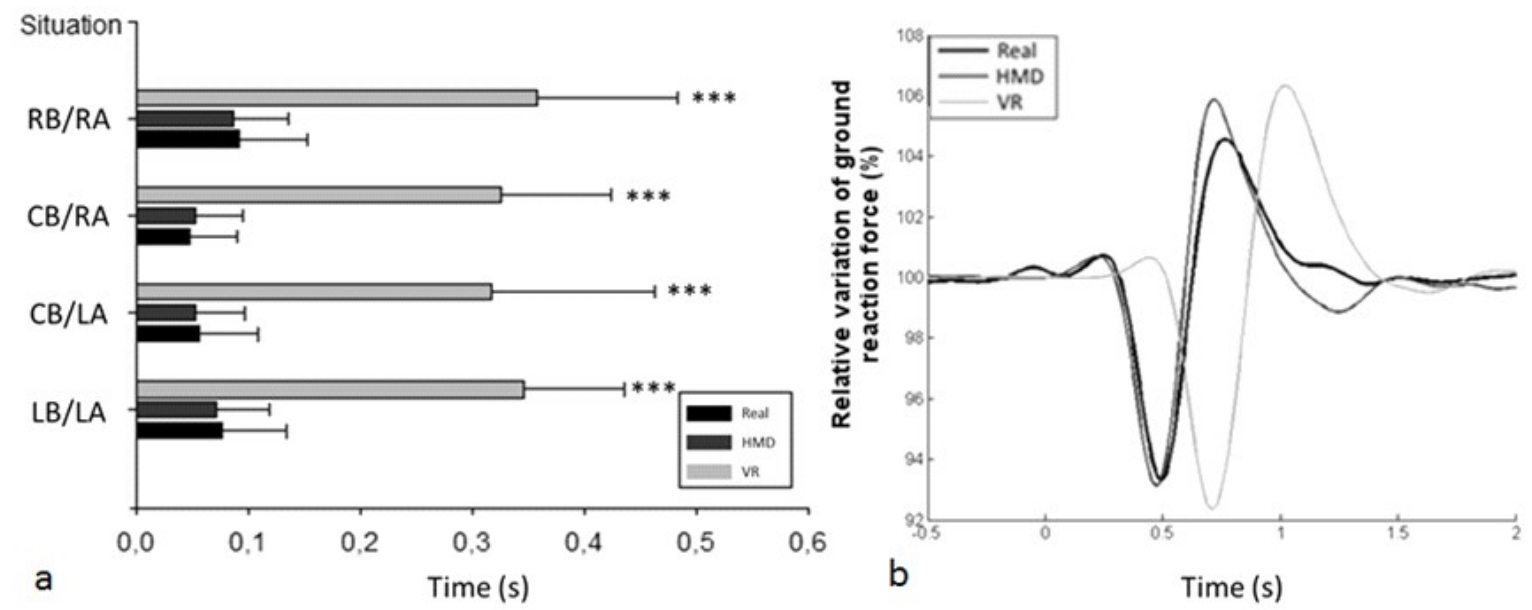

Figure 2: a) Time of first variation of the center of pressure (COP) for the 4 conditions: right ball released with right side avoidance $(R B / R A)$, centered ball released with right side avoidance $(C B / R A)$ and with left side avoidance $(C D / L A)$ and left ball released with left side avoidance (LB/LA). b) Norm of ground reaction forces, expressed in percentage of body weight.

The same kind of results was found concerning the amplitude of the reaction. Figure $2 b$ shows that the subject's behavior is similar, that the Anticipatory Postural Adjustment is preserved in VR but with a small increase of amplitude compared to real situations. When considering the peak values of the ground reaction force, this difference is significant as illustrated in figure $3 a$. It is also the case in the CoP mediolateral displacement (figure 3b). 


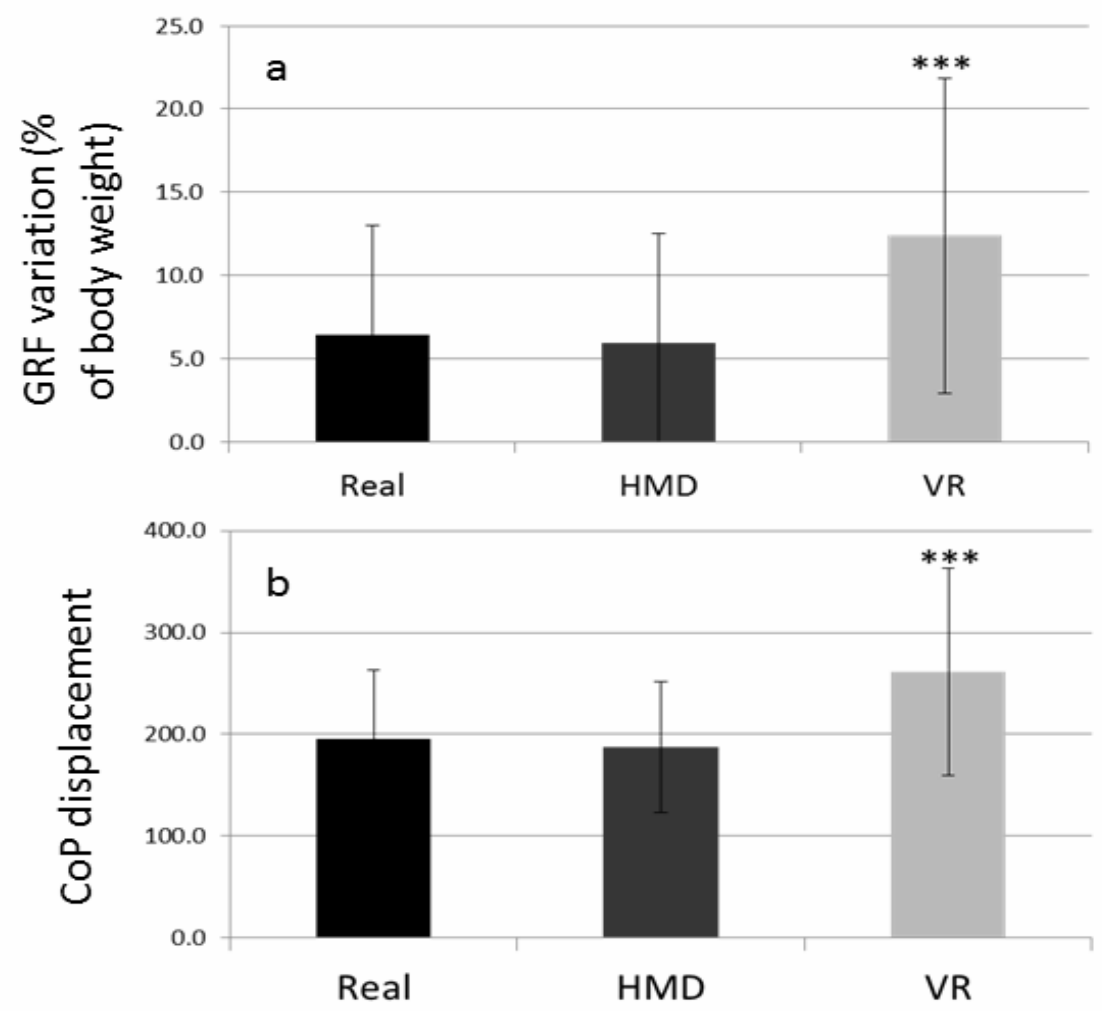

Figure 3: Mean variations for GRF and COP mediolateral displacement for all situations. ${ }^{* * *}$ stand for significant statistical differences $(p<0.001)$.

The results of this experiment provided two main information. First, there is no significant difference between the first two situations in real, with or without HMD. Wearing an HMD device is not disturbing enough to modify the performance of the subjects. Second, although the subject's behavior is similar in real and in VR, the reaction of the subject in VR is overstated and delayed compared to real situations. This experiment emphasizes the importance to take the limits of VR into account. Before reviewing the papers that worked on balance assessment and rehabilitation, let first consider the works that evaluated VR for that purpose.

\section{Evaluation of VR for balance assessment and rehabilitation}

Before exploring the works that used VR for balance assessment and rehabilitation, let us first consider the papers which evaluate VR for that purpose.

Without any external perturbation, Horlings et al. have compared the stability of patients between real and virtual situations in quiet stance. Seventeen young subjects performed four tasks (standing with feet close together or tandem stance on firm and foam surfaces for 60s) under three visual conditions: eyes open without VR, eyes closed, or while viewing a virtual reality scene which moved with body movements [10]. Their results showed that VR causes an increase in postural sway in amplitude similar to that caused by closing the eyes. This increased sway was present irrespective of stance surface, but was greatest on foam. This work tends to demonstrate that stability is not similar between real and virtual environments. Contrary to the work presented above, the experimental conditions weren't the same in both environments, since the subject didn't wear any HMD in the real situation. Nevertheless, the HMD device was a light one, it can thus explain that the results were similar: a larger movement in VR than in real. However, in these two studies, the small field of view of the HMD devices ( $28^{\circ}$ in this study and $36^{\circ}$ in the case study) has certainly changed the peripheral vision of the patient that is very important in balance stability $[11,12]$. It could be interesting to have the same study with a new generation of HMD such as the Oculus Rift. 
The same kind of comparison has been done in rehabilitation. Meldrum et al. indeed compared the effectiveness of a conventional and a virtual-reality based vestibular rehabilitations [13a, 14]. The VR-based training protocol was based on NWFP (Nintendo Wii Fit Plus) which showed high levels of usability and enjoyment with no serious adverse effects $[15,13 \mathrm{~b}]$. The results showed that virtual reality system is more effective than conventional rehabilitation of unilateral peripheral vestibular loss [13a]. In another paper, they concluded that both protocols improve subjects' performances without any significant difference between the two, except in terms of pleasure that subjects had following the VR-based rehabilitation [14].

Cikaljo et al. has evaluated the use of VR for balance telerehabilitation and found the same results [16]. Patients with stroke performed balance training 3 weeks, 2 weeks in the clinical settings and 1 week in the home environment, five times a week, and each time for up to 20 minutes. The results showed that the telerehabilitation approach in VR improved balance in stroke patients and had similar effect on patients' postural functional improvement as conventional balance training in clinical settings.

These works showed that VR was useful for balance rehabilitation because it provides similar progress while adding motivation with a fun training tool. Nevertheless, the results are less positive for balance assessment. The patients immersed in VR indeed made larger displacements than in real. It is problematic if the comparison with real data is done but it can still be useful when comparing the patients in the same condition (all in VR). The impact of VR thus depends on the type of study. Let us first consider the studies on VR for balance assessment and then those on VR for balance rehabilitation.

As illustrated in the case study presented above, assessing balance can be done in VR thanks to its complete control of the stimuli presented to the patients. Amongst these stimuli, the vision is the easiest to control. Several authors have thus proposed to modify the visual information displayed to evaluate their influence on posture control. More accurately, several authors have proposed to use visual controlled stimuli such as a 3D tunnel with different properties (size, frequential or linear movements, etc.). Piponnier et al. worked on the importance of visual and peripheral visions on posture control [11]. 19 healthy young adults were immersed in front of a 3D tunnel, either static or moving sinusoidally in the anterior-posterior direction. Nine visual field conditions were proposed: four central conditions $\left(4,7,15\right.$, and $\left.30^{\circ}\right)$; four peripheral conditions with central occlusions of $4,7,15$, and $30^{\circ}$; and a full visual field condition. The results showed that, in a static environment, the contribution of the visual system in postural control is invariant, regardless of the part of the visual field stimulated. On the opposite, when the optic flow experienced with linear motion, the results suggested that peripheral vision plays a greater role on posture control. Greffou et al. made similar experiment with the same virtual environment to evaluate the influence of the frequency of oscillation of the tunnel on balance depending on the age of the patients [17, 18]. The results showed that younger than 16 year-old children's rely mainly on vision to control their posture. Balance stabilization is then performed between 16 and 19 years, before decreasing after 65 years.

Eikema et al. also studied the influence of age on the postural control but regarding the modulation of sensory reweighting [19]. Young and elderly patients stood on a force plate under two conditions: quiet standing and standing while anticipating randomly approaching virtual objects to be avoided. The visual surrounding was removed or degraded every 60 s to evoke sensory reweighting processes. The results showed that in quiet standing, elderly patients had greater sway variability and were more severely affected by the removal or degradation of visual surround information. Nevertheless, during visual anticipation, the sway variability was not different between the age groups and they were similarly affected by the degradation or removal of the visual surround. 
These results are in accordance with the works above since the mean age of the elderly population was 71.5 years.

To study the influence of vision on balance control in VR, some authors proposed to determine the best visual stimuli that more destabilize the patient. Tossavainen et al. immersed 22 patients in virtual environment thanks to HMD devices and measured their sway with a force plate [20]. The baseline values were acquired without stimulation in three configurations: eyes open, eyes closed and wearing a HMD without stimulus. Three virtual environments were displayed: a 3D oscillating tunnel, a rotating cylinder, and oscillating and rotating dots. The results showed that the 3D tunnel was the most destabilizing stimulus while the cylinder was more efficient on certain people. They open interesting perspectives since different virtual environments could thus be proposed to differentiate pathological patients from normal ones, and perhaps quantify the importance of the pathology.

Since VR allows the complete control of the stimuli presented to the patients, several authors proposed to go further and to study sensory conflicts in order to evaluate balance assessment. Following the work of Akiduki et al. [21], Nishiike et al. examined the effects of sensory inputs of visual-vestibulosomatosensory conflict induced by VR on postural stability [22]. Two different VR conditions were presented. In the control condition, subjects walked voluntarily with the background images of interactive computer graphics proportionally synchronized to their walking pace. In the conflict condition, subjects kept still, but the background images that subjects experienced in the control condition were presented. The results suggested that the conflict condition induced motion sickness, resulting in postural instability. They also suggested that adaptation to the conflict condition decreases the contribution of visual inputs to postural control with re-weighting of vestibulosomatosensory inputs. VR may thus be used as a rehabilitation tool for dizzy patients by its ability to induce sensory re-weighting of postural control. Nevertheless, this study also shows the importance of knowing the latency of the system and being able to minimize it.

Still on the sensory conflict, Keshner at al. have placed healthy adults and adults with bilateral labyrinthine deficiency in a postural stabilization task [23]. They were standing on a support surface that was constantly translating with a $0.25 \mathrm{~Hz}$ sinusoid. On the contrary, the displacement of the visual environment was varied in direction and frequency. To evaluate the postural stabilization, they made a kinematical analysis of the posture. Their results confirmed that visual input is an important component of stabilization. They also exhibited that the reaction to the amplitude of virtual environment displacement was related to the availability of vestibular information. The authors concluded that VR can support both diagnosis and rehabilitative training of individuals with sensory integration impairments. Keshner et al. also used VR to assess the ability of healthy adults and adults with labyrinthine deficit to react to a loss of balance [24]. To this end, they proposed three input configurations: the force plate supporting the patient is translated, or the virtual world is moved, or both are translated. The results showed that when there is a confluence of meaningful inputs, none of the inputs are suppressed in healthy adults; the postural response is modulated by all existing sensory signals in a non-additive fashion. Labyrinthine deficient adults suppress visual inputs.

Bugnariu et al. also studied conflicting visual and somatosensory stimuli but on young and old adults [25]. They analyzed concurrently the center of pressure (CoP), the center of mass and the muscular activity thanks to an EMG system. Four perturbations were proposed: visual-only and surface-only (only the virtual environment or the supporting surface was tilted), concordant and discordant (visual and surface perturbations moved synchronously in the same or opposite direction). The results showed globally that the visual-only perturbation elicits minimal postural responses compared to others, suggesting that the information is weighted more in regulating upright posture. Nevertheless, as for the study of Greffou et al. $[17,18]$, aging influences stance recovery, especially in the presence of sensory conflicts.

All these works enhance that VR provides an infinite number of configurations, combining visual, haptic and/or audio inputs that can be used for balance assessment and rehabilitation. For each of these inputs, the stimuli can be very varied but are always controllable and reproducible. For visual input for example, the stimulus can be a 3D tunnel, dots... The design of balance assessment tools is then not limited by technology and only requires to find the best stimuli in accordance to the pathology of the studied population. 
Concerning the disadvantages of VR, these works have shown the predominance of visual input and the importance to take care of the conflict between the senses. It is thus important to minimize the latency at best. Nevertheless, all these studies used the same input stimuli for the patients and the comparison was made between groups of people. The latency is thus not a problem anymore since their influence is the same for everyone. This drawback is then annihilated by the ability of VR to control all the stimuli and to propose a reproducible environment. Concerning the distance perception, no work is dealing with it as it has been done in other domains such as for locomotion assessment [26-28]. The distance of the virtual environment used for balance assessment or rehabilitation is often small so it can less influence the perception in VR nevertheless it would be interesting to validate this statement.

\section{$5 \quad$ VR for balance rehabilitation}

As suggested by the studies described in the previous section, VR has all the advantages to be used as a training tool for balance rehabilitation. Nevertheless, VR embeds a large number of software and hardware technologies. The choice of these technologies has an influence on the degree of immersion and presence of the patients and thus the potential effectiveness of the system for rehabilitation. Furthermore, this choice has also consequences on the information perceived. For instance, the depth information cannot be well perceived without stereoscopic vision and not all the visual information can be picked up without being able to change viewpoint such as with a head tracking system. These devices can thus modify the perception of distance as discussed above. In this section, we thus review the papers according to their technological choices.

The easiest display that can be used in a VR system is a flat screen without any stereoscopic vision. Many authors used such a system in balance rehabilitation, adding an input device that allows the patient to control a virtual environment by weight shifting, rotation or inclination of his body. The first input device used is a force platform or a balance board. It records the center of pressure and potentially the force applied on the board and transforms those data as an input for the movement in the virtual environment.

Several authors worked on the rehabilitation of people with Parkinson disease or elderly people. The studies rely on the weight shifting of the patient, either on a balance board $[29,30]$ or on an inclinable force platform [31, 32]. Yen and al. used the CoP of the patient to shift a virtual character in a classical environment or to incline a virtual plate containing balls that roll until they fall in a hole. After several weeks of training, a comparison is made between the improvement of a control group and the VR group to quantify the added value of VR. They conclude that both training are equivalent, only the prevention of risk of falls would be more effective in sensory and visual reduced conditions with VR. In the same way, Kosse and al. provided a maze inside which balls are moving, controlled in the same manner. Improvements of the subjects are measured by BBS or figure of eight after and before the therapy. Mendes and al. and Llorens and al. did not create a specific virtual environment but selected some of those available in the Wii Fit device, requiring multidirectional shifts, alternating steps or stationary control of the player's center of mass and trained the subjects for several weeks. The evaluation of the improvement is done either with the reach test (assesses limits of stability in the standing position as the maximum distance a subject can reach without moving his feet from the floor) or with different classical parameters (BBS, ART, ST...).

Another way to exploit weight shifting is to use specialized devices equipped with sensors. For example, Cikaljo and al. have placed stroke subjects [33] in an apparatus made of aluminum and wood, preventing the subject from falling and enabling him to move a virtual character by weight shifting. The character must travel across a path full of obstacles as fast as possible. The level can be changed by modifying the number of obstacles. They quantified the evolution of the subject thanks to the BBS before and after therapy but also considering the travel time of the virtual character. Jeong and al. also created a specific object to develop balance rehabilitation of stroke subjects. The patient has to travel across a virtual road on a bike, following the virtual central road line while keeping his CoP stable [34]. 
Finally, the last set of works that used VR without stereoscopic vision is based on Motion Capture systems. These systems can be based on optical, inertial or depth sensors. They go from a simple Microsoft Kinect up to a complete optoelectronic system accurate at less than $1 \mathrm{~mm}$.

Llorens and al. used a Microsoft Kinect and a screen (TV or LCD screen) to study the rehabilitation of patients suffering from residual hemiparesis after stroke [35]. The patients were divided into 2 groups, one is trained at home and the other in a clinical environment. The virtual environment is made up of an empty room with a checkered floor and various items appearing all around the virtual subject. The level of the exercise can be changed by modifying the size and distance of objects, or their appearance duration. They had to touch items with their closest foot. The therapy lasted 8 weeks and the balance was estimated before and after rehabilitation with BBS. The results showed that VR-based in-clinic and VR-based telerehabilitation interventions offer both similar and significant improvement of the patients. Everding and al. allowed 3 service members with upper and/or lower extremity amputations to guide a virtual boat thanks to markers placed above the pelvis that are captured with a motion capture system (Vicon Inc., Oxford, United Kingdom) [36]. To better immerse the subject and enable different levels of difficulty, subjects stand on a moving platform that simulate the waves' movement. Performance is evaluated through the travel time of the boat. In a similar manner, Hawkins and al, Barton and al. immerse healthy subjects and children with CP diplegia in a fantastic virtual environment, made of flying carpet or dragons that move simultaneously with markers placed on the pelvis and the trunk of the subjects. The latter must catch virtual items placed on the virtual environment to accelerate their displacement. Moreover, different initial positions can be tested (kneel sitting, high kneeling, standing) to modify the difficulty of the task [3739].

To increase the presence of immersed patients, VR can be used combined to stereoscopic vision. Nevertheless, this setup is more complex and requires more expensive devices. It comes from a flat screen and stereoscopic glasses up to a CAVE, a virtual environment composed of several walls surrounding the patient.

Llorens and al. used a panoramic screen and stereoscopic glasses to immerse patients with brain injury in a virtual environment where they have to reach virtual objects more or less far from them. They called their low-cost system BioTrak. Each subject is represented by a simple and intuitive avatar they can see from a third-person viewpoint. The movement of the subject was captured with any tracking system and many parameters can be modified to adapt the difficulty of the simulation to the subject's pathology and morphology (resting time of the subject, number of repetitions, distance to the items, time they remain visible, size...) [40]. All patients trained for 20 sessions of 20 minutes with more and more difficult exercises depending on their improvement. Balance was assessed at the beginning, at the end and one month after the end of the therapy by BBS and POMA, but also using a NEDSVE/IBV dynamometric platform which combines static posturography assessment with dynamic tests and provides a global index evaluating the balance. No control group was used, but still the results showed some significant improvements between both initial and final balance measures, and gains remains a month after the therapy. According to the subjects, Biotrack insures high degrees of presence, immersion and user-friendliness.

Kim and al. used the same protocol as [34] but with stereoscopic vision thanks to an HMD device [41]. The subjects were on a bike and had to follow the central line while keeping their CoP as stable as possible. Both showed that training improved balance, since subjects managed better and faster the exercise after weeks of training. However, both used a fixed bicycle, leading to a conflictual sensory input (no tilting even though the virtual bicycle is moving).

Yeh and al. made patients suffering from vestibular dysfunction and healthy patients follow a rehabilitation treatment with stereoscopic glasses [42]. He uses a Microsoft Kinect device to capture the subject's movement, and a Nintendo Wii Fit to get the statokinesigram of each patient. They extracted several parameters from it which describe at best the balance of the subject during various VR exercises (based on eyeball movement, head movement, waist rotation movements, body rotation movements with eyes closed and ball throwing test). 
The objective is to automatically classify the subjects in 2 classes: healthy and unhealthy. They uses Support Vector Machine (SVM) since the variations of parameters are very different according to whether the subject is healthy or not. This emphasizes the efficiency of the rehabilitation tool for their patients. Future works could extend the analysis to automatically quantify the degree of pathology of a patient.

All these studies confirm that VR can be a balance rehabilitation tool. The evaluation of the ability of the patients is done before and after the rehabilitation. It means that contribution of VR to the patients' stability and well-being is assessed globally and is successful whatever the limits of VR.

\section{Conclusion}

VR is now commonly used in many domains and especially in the medical field. Balance assessment and rehabilitation are also taking advantage of the standardized, reproducible and controllable features of VR. Nevertheless, VR is based on software and hardware that computes the virtual environment and renders it. It means that it cannot provide a perfectly realistic environment but more problematic it can modify the perception and the interaction with this virtual environment. VR has thus also two main limitations that are sometimes omitted or understated. The latency, the delay between the input devices (action of the immersed patient) and the output devices (reaction of the virtual environment and thus the change of the stimuli presented to the patient), is the first important limit of VR. Concerning balance assessment, this delay creates a sensory conflict that can lead to sickness or fall. Moreover, when dealing with reaction times, for instance to evaluate the sway velocity or the anticipatory postural adaptation of a patient who avoids a virtual projectile, the sensors equipped on the patient must be synchronized with the launch of the virtual projectile. It is technologically difficult to setup and the results strongly depend on the latency of the system since the reaction of the patient is added to the delay between the computation of the ball and the perception of the visual stimulus. Latency must then be taken into account when the results must be compared to real data from the literature for instance. The second limit of VR is the underestimation of perceived distance in virtual environment compared to real situation. The problem again is when comparing parameters of the patients' performance in real and in virtual. In this review, we described a case study to emphasize the advantages and limits of VR for balance assessment and rehabilitation. The results showed a temporal shift of the data and a bigger displacement of the CoP. It could be interesting to determine the relative influence of latency and perception of distance in the alteration of the data and to quantify the minimum acceptable thresholds. In the context of balance rehabilitation, the studies are usually comparing data before and after the rehabilitation. VR is then considered as a training tool and the goal is to evaluate the evolution of the patient. The limits are then not problematic since they are the same before and after the rehabilitation.

To conclude, many studies used VR for balance assessment and rehabilitation. The ability to control all the parameters of the simulation offers an infinite number of configurations. It allows an accurate evaluation of each factor responsible of balance or its loss but also the comparison of the relative influence of each sense thanks to sensory conflicts studies. Finally, it provides a new generation of rehabilitation tools and at the same time the way to assess the efficiency of these tools to optimize them.

A very large majority of papers use the visual perception and the proprioception in their studies. VR can have various input and output devices such as haptic, sound, etc. For instance, Milosevic and McConville used the combination of visual and auditory feedbacks [43] to evaluate postural control. A lot of studies can be made to go further and evaluate the contribution of more complex VR systems. Adding real time feedbacks can complete the spectrum of the features available. Nevertheless, it requires more engineering skills because of the complexity of the setups. A multidisciplinary approach will open several years of promising studies in the field of balance assessment and rehabilitation. 
[1] Levac D, Espy D, Fox E, Pradhan S and Deutsch JE. "Kinect-ing" With Clinicians: A Knowledge Translation Resource to Support Decision Making About Video Game Use in Rehabilitation. Physical Therapy, 95(3): 426-440, March 2015.

[2] Hamilton EC, Scott DJ, Fleming JB, Rege RV, Laycock R, Bergen PC and al. Comparison of video trainer and virtual reality training systems on acquisition of laparoscopic skills. Surgical Endoscopy And Other Interventional Techniques, 16(3): 406-411, March 2002.

[3] Bideau B, Kulpa R, Vignais N, Brault S, Multon F and Craig C. Using Virtual Reality to Analyze Sports Performance. IEEE Computer Graphics and Applications, 30(2): 14-21. DOI:10.1109/MCG.2009.134, 2010.

[4] Sanchez-Vives M and Slater M. Opinion: From presence to consciousness through virtual reality. Nature Reviews Neuroscience, 6(4): 332-339, April 2005.

[5] Slater M, Khanna P, Mortensen J, and Yu I. Visual Realism Enhances Realistic Response in an Immersive Virtual Environment. IEEE Computer Graphics and Applications, 29(3): 76-84, May 2009.

[6] Hendrix C, Barfield W. Presence within virtual environments as a function of visual display parameters. Presence: Teleoperators and Virtual Environments, 5 (3): 274-289, 1996.

[7] Tarr M and Warren W. Virtual reality in behavioral neuroscience and beyond. Nature Neuroscience, 5(Supp): 1089-1092, November 2002.

[8] Slater $M$, Linakis $V$, Usoh $M$ and Kooper R. Immersion, presence and performance in virtual environments: An experimental with tri-dimensional chess. In M. Green (Ed.), Virtual reality software and technology (pp. 163-172). Hong Kong, ACM Press, 1996.

[9] Bideau B, Kulpa R, Ménardais S, Fradet L, Multon F, Delamarche P, and al. Real Handball Goalkeeper vs. Virtual Handball Thrower. Presence: Teleoperators and Virtual Environments, 12(4): 411-421, August 2003.

[10] Horlings C, Carpenter MG, Küng UM, Honegger F, Wiederhold B, and Allum J. Inuence of virtual reality on postural stability during movements of quiet stance. Neuroscience Letters, 451(3): 227-231, February 2009.

[11] Piponnier JC, Hanssens JM, and Faubert J. Effect of visual field locus and oscillation frequencies on posture control in an ecological environment. Journal of Vision, 9(1): 13-13, January 2009

[12] Hanssens JM, Piponnier JC, and Faubert J. Influence of central and peripheral visual field on the postural control when viewing an optic flow stimulus. Journal of Vision, 8(6): 858-858, April 2010.

[13a] Meldrum D, Herdman S, Moloney R, Murray D, Duy D, Malone K and al. Effectiveness of conventional versus virtual reality based vestibular rehabilitation in the treatment of dizziness, gait and balance impairment in adults with unilateral peripheral vestibular loss: a randomised controlled trial. BMC Ear, Nose and Throat Disorders, 12(1):3, 2012.

[13b] Meldrum D, Glennon A, Herdman S, Murray D, and McConn-Walsh R. Virtual reality rehabilitation of balance: assessment of the usability of the Nintendo Wii R Fit Plus. Disability and Rehabilitation: Assistive Technology, 7(3): 205-210, May 2012.

[14] Meldrum D, Herdman S, Vance R, Murray D, Malone K, Duy D and al. Effectiveness of conventional versus virtual reality-based balance exercises in vestibular rehabilitation for unilateral peripheral vestibular loss: results of a randomised controlled trial. Archives of Physical Medicine and Rehabilitation, April 2015. 
[15] Virtual reality rehabilitation of balance: assessment of the usability of the Nintendo Wii $\left(^{\oplus}\right)$ Fit Plus. Dara Meldrum Aine Glennon Susan Herdman Deirdre Murray Rory McConn-Walsh Disability and rehabilitation. Assistive technology, 2011.

[16] Cikajlo I, Rudolf M, Goljar N, Burger H, and Matjafic Z. Telerehabilitation using virtual reality task can improve balance in patients with stroke. Disability and Rehabilitation, 34(1): 13-18, January 2012.

[17] Greffou S, Bertone A, Hanssens J-M and Faubert J. Development of visually driven postural reactivity: A fully immersive virtual reality study. Journal of Vision, 8(11):15, 2008

[18] Greffou S and Faubert J. Life-span study of visually driven postural reactivity: A fully immersive virtual reality approach. Journal of Vision, 8(6):426, 2008

[19] Eikema D, Hatzitaki V, Tzovaras D, and Papaxanthis C. Age-dependent modulation of sensory reweighting for controlling posture in a dynamic virtual environment. AGE, 34(6): 1381-1392, December 2012.

[20] Tossavainen T, Juhola M, Pyykkö I, Aalto H, and Toppila E. Development of virtual reality stimuli for force platform posturography. International Journal of Medical Informatics, 70(2-3): 277-283, July 2003.

[21] Akiduki H, Nishiike S, Watanabe H, Matsuoka K, Kubo T, and Takeda N. Visual-vestibular conflict induced by virtual reality in humans. Neuroscience Letters, 340(3): 197-200, April 2003.

[22] Suetaka Nishiike, Suzuyo Okazaki, Hiroshi Watanabe, Hironori Akizuki, Takao Imai, Atsuhiko Uno, Tadashi Kitahara, Arata Horii, Noriaki Takeda, and Hidenori Inohara. The effect of visualvestibulosomatosensory conflict induced by virtual reality on postural stability in humans. The Journal of Medical Investigation, 60(3.4): 236-239, 2013.

[23] Keshner EA and Kenyon RV. Influences on Postural Orientation in a Virtual Environment. pages 13071312. IEEE, May 2007.

[24] Keshner EA, Kenyon RV, and Dhaher Y. Postural research and rehabilitation in an immersive virtual environment. volume 4, pages 4862-4865. IEEE, 2004.

[25] Bugnariu $\mathrm{N}$ and Fung J. Aging and selective sensorimotor strategies in the regulation of upright balance. Journal of NeuroEngineering and Rehabilitation, 4(1):19, 2007.

[26] Gérin-Lajoie M, Richards CL, Fung J, and McFadyen BJ. Characteristics of personal space during obstacle circumvention in physical and virtual environments. Gait \& Posture, 27(2): 239-247, February 2008.

[28] Jones A, Swan JE, Singh G, and Kolstad E. The Effects of Virtual Reality, Augmented Reality, and Motion Parallax on Egocentric Depth Perception. pages 267-268. IEEE, 2008.

[29] Mendes F, Pompeu JE, Lobo AM, da Silva KG, de Paula Oliveira T, Zomignani AP and al. Motor learning, retention and transfer after virtual-reality-based training in Parkinson's disease effect of motor and cognitive demands of games: a longitudinal, controlled clinical study. Physiotherapy, 98(3): 217-223, September 2012.

[30] Lloréns R, Albiol S, Gil-Gómez J, Alcañiz M, Colomer C, and Noé E. Balance rehabilitation using custommade Wii Balance Board exercises: clinical effectiveness and maintenance of gains in an acquired brain injury population. International Journal on Disability and Human Development, 13(3), January 2014.

[31] Yen CY, Lin KH, Hu MH, Wu RM, Lu TW, and Lin CH. Effects of Virtual Reality-Augmented Balance Training on Sensory Organization and Attentional Demand for Postural Control in People With Parkinson Disease: A Randomized Controlled Trial. Physical Therapy, 91(6): 862-874, June 2011.

[32] Kosse NM, Caljouw SR, Vuijk P, and Lamoth C. ExergamIng: Interactive Balance tralnIng in healthy community dwelling older adults. Journal of Cybertherapy \& Rehabilitation, 2011.

[33] Cikajlo I, Rudolf M, Goljar N, and Matjacic Z. Virtual reality task for telerehabilitation dynamic balance training in stroke subjects. pages 121-125. IEEE, June 2009. 
[34] Jeong SH, Piao YJ, Chong WS, Kim YY, Lee SM, Kwon TK and al. The Development of a New Training System for Improving Equilibrium Sense Using a Virtual Bicycle Simulator. pages 2567-2570. IEEE, 2005.

[35] Lloréns R, Noé E, Colomer C, and Alcañiz M. Effectiveness, Usability, and Cost-Benefit of a Virtual Reality Based Telerehabilitation Program for Balance Recovery After Stroke: A Randomized Controlled Trial. Archives of Physical Medicine and Rehabilitation, 96(3): 418-425, March 2015.

[36] Everding VQ and Kruger SE. Virtual reality enhanced balance training for service members with amputations. pages 12. IEEE, June 2011.

[37] Hawkins P, Hawken $M$ and Barton G. Effect of game speed and surface perturbations on postural control in a virtual environment. In Proc. of the 7th ICDVRAT, 311-318, 2008.

[38] Barton GJ, Hawken MB, Foster RJ, Holmes G, and Butler PB. Playing the Goblin Post Oce game improves movement control of the core: A case study. pages 15. IEEE, June 2011.

[39] Barton GJ, Hawken MB, Foster RJ, Holmes G, and Butler PB. The effects of virtual reality game training on trunk to pelvis coupling in a child with cerebral palsy. Journal of NeuroEngineering and Rehabilitation, 10(1): 15, 2013.

[40] Lloréns R, Colomer-Font C, Alcañiz M, and Noé-Sebastián E. BioTrak virtual reality system: Effectiveness and satisfaction analysis for balance rehabilitation in patients with brain injury. Neurología (English Edition), 28(5): 268-275, June 2013.

[41] Kim NG, Yoo CK, and Im JJ. A new rehabilitation training system for postural balance control using virtual reality technology. IEEE Transactions on Rehabilitation Engineering, 7(4):482-485, December 1999.

[42] Yeh SH, Huang MC, Wang PC, Fang TY, Su MC, Tsai PY, and Rizzo AA. Machine learning-based assessment tool for imbalance and vestibular dysfunction with virtual reality rehabilitation system. Computer Methods and Programs in Biomedicine, 116(3): 311-318, 2014

[43] Milosevic M, and McConville K. Audio-visual biofeedback system for postural control. International Journal on Disability and Human Development, 10(4), pp. 321-324, 2011. 